\title{
Occam's Inversion and the North American Central Plains Electrical Anomaly
}

\author{
Catherine deGroot-Hedlin and Steven Constable \\ Institute of Geophysics and Planetary Physics, La Jolla, CA92093-0225, U.S.A.
}

(Received March 3, 1993; Revised July 29, 1993; Accepted September 3, 1993)

\begin{abstract}
The COPROD2 magnetotelluric (MT) data set obtained over the North American central plains conductivity anomaly in the Canadian shield features a predominantly one-dimensional response at periods shorter than $14 \mathrm{~s}$ and a predominantly two-dimensional response at longer periods (to $910 \mathrm{~s}$ ). A subset of the COPROD2 data consisting of both strike-parallel and strikeperpendicular components of the MT apparent resistivity and phase data at periods between 14-910 s was subjected to the Occam's inversion process, which attempts to find maximally smooth models which fit a data set to a specified misfit. After 9 iterations a model was found fitting these data to $10 \%$ in resistivity and $2.9^{\circ}$ in phase, and after a few more iterations excess structure was removed to reveal three discrete conductive zones of resistivity less than $1 \Omega \mathrm{m}$ at depths of 8-22 km in a relatively resistive background layer of $100-1000 \Omega \mathrm{m}$. The inversion algorithm was modified to take advantage of the 1D structure of the shorter periods, which were inverted to obtain a model representative of surface sediments which extend to a depth of about $2 \mathrm{~km}$. This surface structure was included in the long-period inversion by imposing a second penalty term in the regularized inversion, and the resulting model featured a broader, more complex conductive anomaly and a pronounced, westward-dipping fabric in the mid- to lower-crustal rocks. Graphitic rocks can account for the highly conductive parts of the models.
\end{abstract}

\section{Introduction}

In this paper we present the results of two-dimensional (2D), smooth inversions of the COPROD2 magnetotelluric (MT) data set (Jones and SAVAGE, 1986; Jones, 1988) using the Occam's inversion algorithm of CONSTABLE et al. (1987), modified for 2D MT by DEGROOT-HEDLIN and Constable (1990). The MT data were obtained over the North American Central Plains (NACP) and Thompson Belt electromagnetic anomalies in order to obtain a quantitative model of features previously identified in numerous geomagnetic depth sounding (GDS) surveys (see the summary by JONES and CRAVEN, 1990). The GDS surveys indicated that the NACP anomaly is a large scale, linear feature extending from southeastern Wyoming northward to the exposed Canadian Shield in northern Saskatchewan, and from there extending eastward along the regional geological strike into the Hudson's Bay basin. The alignment of zones of intense shearing and folding in the exposed Precambrian crust with both the northern and southern ends of the NACP anomaly led CAMField and Gough (1977) to suggest that the anomaly is associated with a Proterozoic plate boundary.

Our aim here is to develop methods of interpretation of approximately 2D MT data sets using smooth inversion methods and to apply these techniques to determine the conductivity structure of the NACP anomaly. Although three-dimensionality is evident along the length of the conductivity anomaly in the GDS data (AlABI et al., 1975), as well as in MT observations recorded in two profiles to the north of the COPROD2 line (JONES and CRAVEN, 1990), the NACP anomaly may be interpreted as a two-dimensional (2D) feature to a first approximation due to its long strike length. 
We provide a brief introduction of the inversion method used to investigate the $2 \mathrm{D}$ conductivity structure of this region, followed by the presentation of a 2D model fitting the low frequency TE and TM apparent resistivity and phase data. A modification of the inversion method is introduced in order to accomodate a special property of the COPROD2 data set; namely, the apparent one dimensionality of the high frequency data. Finally, another modification to the Occam method is introduced to force smoothing between fixed or preferred structure and the free parameter part of the model. This approach is used to create a model which simultaneously fits both high and low frequency COPROD2 data.

\section{Inversion Method}

The 'Occam' process (CONSTABLE et al., 1987) is an approach to the regularization of nonlinear inversion problems, particularly those involving electromagnetic forward solutions, which are almost always nonlinear. Regularization refers to the application of a penalty on the model; the penalty is minimized subject to the constraint that the model fit the observed data. Here we apply a penalty to the magnitude of the first derivative of the model resistivities (the 'roughness'), which results in a smooth-looking model (the smoothest by this particular measure; there are other measures of roughness, such as the magnitude of the second derivative). A smoothing penalty is useful in electrical methodology, because generally the true least-squares solution produces models with physically unrealistic conductivity contrasts (e.g. PARKER, 1980, 1984). Any attempt to attain the least-squares solution using approximate or iterative methods tends to these extremes if the model parameterization allows, introducing spurious structure into the model. Regularization avoids these extremes if the data fit is relaxed slightly from the leastsquares value, and one hopes that conductivity contrasts appearing in the models are likely to exist in the Earth as well, albeit in a less smooth form. However, it must be emphasized that smooth models are not the final step in interpretation; they are merely the simplest in some specific, mathematical sense, and not necessarily the most geologically realistic.

Application of the Occam process to 2D magnetotelluric data is described in DEGROOTHedlin and Constable (1990). We review the mathematical notation briefly because the technique is modified later in this paper. We minimize an unconstrained functional

$$
U=\|\partial \mathbf{m}\|^{2}+\mu^{-1}\|\mathbf{W}(\mathbf{d}-F(\mathbf{m}))\|^{2}
$$

where $\boldsymbol{\partial}$ is a matrix operator that takes first differences of the elements in the model vector $\mathbf{m}$ and the second term is the standard 2-norm, or sum of squares, measure of misfit between the data, $\mathbf{d}$, and the nonlinear forward functionals which act upon the model to produce model response, $F$. The matrix $\mathbf{W}$ is a covariance weighting operator, which in practice means a diagonal matrix of inverse data errors. A Lagrange multiplier $\mu^{-1}$ trades off roughness against data fit, and will be chosen to achieve an adequate, or barely adequate, fit to the data. We minimize (1) by differentiating $U$ with respect to the model and setting the result equal to zero. With a little re-arrangement this yields a term for a new model based on a linearization about an initial model:

$$
\mathbf{m}(\mu)=\left[\mu \partial^{T} \boldsymbol{\partial}+(\mathbf{W J})^{T} \mathbf{W J}\right]^{-1}(\mathbf{W J})^{T} \mathbf{W} \hat{\mathbf{d}}
$$

where $\mathbf{J}$ (the Jacobian matrix) is the matrix of partial derivatives of $F$ with respect to the initial model $\mathbf{m}_{o}$ and $\hat{\mathbf{d}}=\mathbf{d}-F\left[\mathbf{m}_{o}\right]+\mathbf{J m}_{o}$ is the data vector offset by two known terms. The Lagrange multiplier is chosen at each iteration using a separate $1 \mathrm{D}$ optimization routine to provide the best misfit or the desired misfit.

The extension of the method to $2 \mathrm{D}$ magnetotellurics in principle only requires an extension of the roughness measure to two dimensions. However, from the inversion's perspective the model 
is still a single 1D vector $\mathbf{m}$. In a Cartesian coordinate system in which $\mathbf{x}$ is parallel to the strike direction and $\mathbf{z}$ is down, the roughness measure may be represented as

$$
\mathbf{R}=\left\|\partial_{y} \mathbf{m}\right\|^{2}+\left\|\partial_{z} \mathbf{m}\right\|^{2}
$$

where $\boldsymbol{\partial}_{y}$ and $\boldsymbol{\partial}_{z}$ are the first difference operators for laterally adjacent, and vertically adjacent blocks, respectively. The ordering of the roughness penalties is unimportant, so equivalently we have

$$
\mathbf{R}=\|\partial \mathbf{m}\|^{2}
$$

where

$$
\boldsymbol{\partial}=\left[\begin{array}{l}
\boldsymbol{\partial}_{y} \\
\boldsymbol{\partial}_{z}
\end{array}\right]
$$

and in practice the penalties for both horizontal and vertical roughness are incorporated this way into a single matrix. Indeed, any row of $\boldsymbol{\partial}_{z}$ or $\boldsymbol{\partial}_{y}$ can be omitted to allow unpenalized boundaries to develop, or additional penalties between non-adjacent parameter blocks added if, for example, they are considered a priori to be geologically equivalent. The size of $\boldsymbol{\partial}$ is immaterial because in the actual inversion it is $\partial^{T} \boldsymbol{\partial}$ that is used (see Eq. (2)).

The method also allows for the simultaneous solution of the static shifts and resistivities which yield the smoothest models (DEGROOT-HEDLIN, 1991). Static shifts in the data are caused by near-surface small-scale conductive inhomogeneities which distort the electric field of regional interest but leave the magnetic field virtually unperturbed. Phase information is unaffected by static distortion, while the apparent resistivities are shifted by a frequency independent multiplicative scale factor. Obviously, inverting data with shifts which vary from station to station will result in models displaying a large degree of lateral roughness. We solve for the smoothest resistivity model but allow the $\log _{10}$ (resistivities) for each mode to vary by a free additive constant. The average resistivity of the model must be fixed in some way, and in this paper we do this by constraining the sum of the scale factors to be zero, on the assumption that static shifts caused by surficial resistive inhomogeneities are balanced by static shifts due to conductive bodies. However, there are various other ways to fix the resistivities of the model, such as fixing the static shift at one station or including fixed or preferred resistivities in the model.

\section{Joint Inversion of the TE and TM COPROD2 Data}

The inversion method is applied only to longer period data for two reasons. Firstly, inclusion of the high frequency data requires a very fine finite element mesh near the surface, which propagates through the vertical nodes throughout the model, considerably increasing both the time and memory required to perform the forward computations. Secondly, for short period data the receiver sites are separated by many skin depths, and the sites may be treated as independent, giving information only on the shallow structure near the site at which they are collected. Structure resolved by short period data at one site has negligible effect on the responses at neighbouring stations. Thus inclusion of short period data into a fully $2 \mathrm{D}$ inversion would have little effect on the $2 \mathrm{D}$ resolution of the shallow structure. In general, the cutoff below which the periods should be excluded from a $2 \mathrm{D}$ or $3 \mathrm{D}$ inversion will depend upon the station spacing and subsurface resistivity. The lower cutoff for the COPROD2 data was chosen to be $14 \mathrm{~s}$, which also coincides with the appearance of the anomalies in the phase data.

A reasonable estimate of errors must be made in order to determine an appropriate level of misfit between the data and model responses. Errors supplied with the COPROD2 data are derived from uncertainty in the MT response function estimation, and should reflect the extent to which repeat experiments at the same locations would yield similar results. Using 

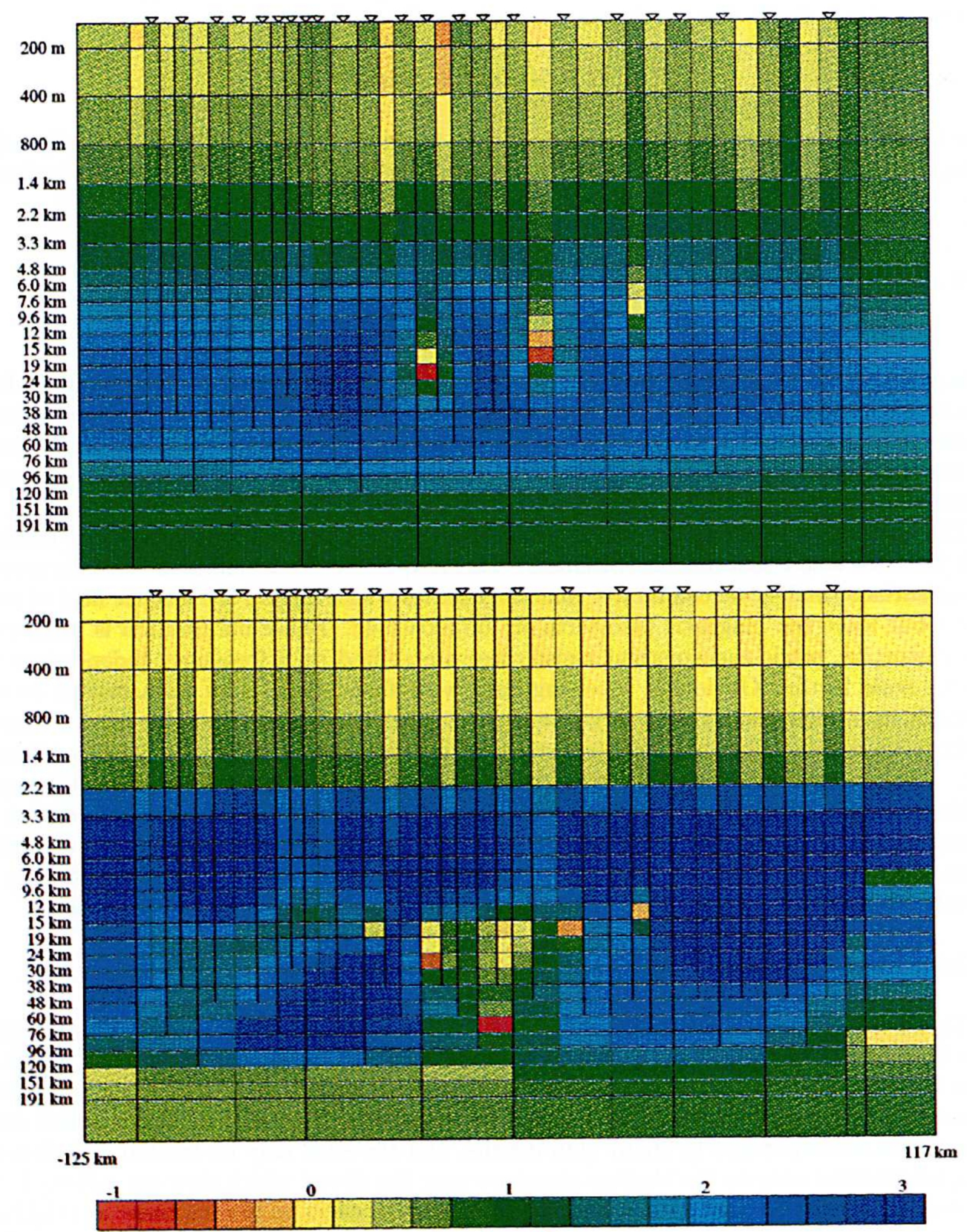

Scake, log resistivity

Fig. 1. Top: A joint TE and TM model for apparent resistivities and phases at 7 periods in the range $14-910 \mathrm{~s}$. The station locations are indicated by the triangles. The distance scale is in kilometres east of $103.25^{\circ} \mathrm{W}$. Conductive structure associated with the NACP anomaly appears as discontinuous anomalies from -50 to 50 $\mathrm{km}$ centred at depths of $8 \mathrm{~km}$ in the east to $22 \mathrm{~km}$ in the west. Bottom: In this model the upper 5 layers are penalized against a surface structure similar to that shown in Fig. 3. The model fits the data to $15 \%$ on average, and shows a persistent westward dipping fabric for the lower crust between about $10 \mathrm{~km}$ and the mantle. 

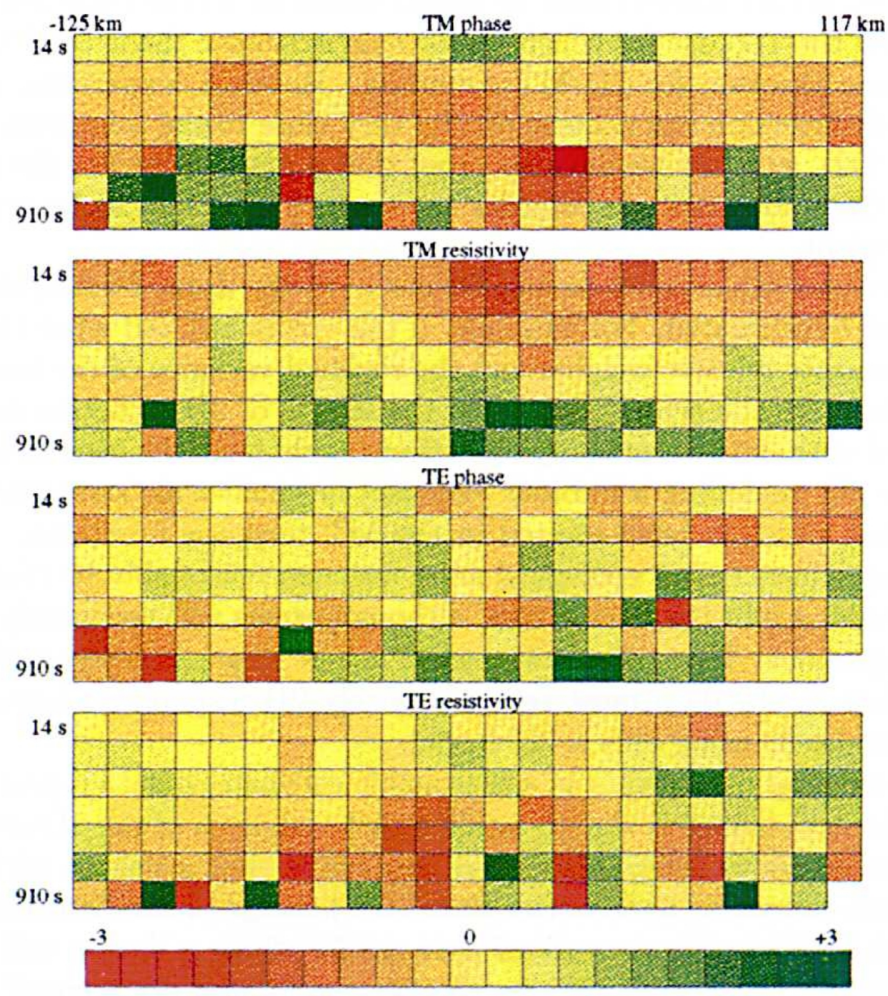

Scale, weighted residual

Fig. 2. Weighted residuals corresponding to the upper model of Fig. 1. Eight points (of a total of 640) are outside the range -3 to 3 . Negative residuals (red) indicate that the data are smaller than the corresponding responses and positive residuals (green) indicate that the responses are smaller. White areas indicate missing data. The horizontal scale corresponds to station number and is monotonic in distance but uneven due to the uneven station distribution.

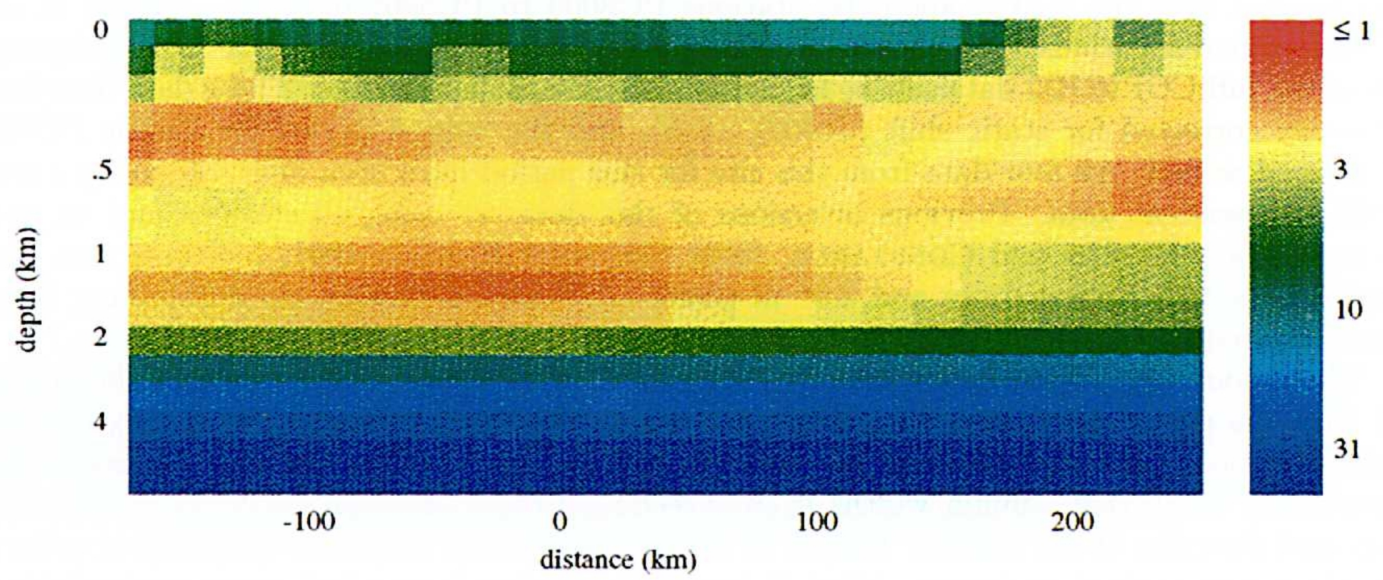

Fig. 3. Pseudo-1D model fitting the data in the range $.056 \mathrm{~s}(18 \mathrm{~Hz})$ to $7.1 \mathrm{~s}$. The model was generated by applying a greater penalty to horizontal roughness than to vertical roughness. The color scale is in $\Omega \mathrm{m}$. 
these error estimates, no 2D model could be found which fit to the expected value of RMS 1.0. Unfortunately, unlike the 1D case for which we have an existence theorem (PARKER, 1980), there is as yet no existence theorem for 2D MT sounding. However, experience with $1 \mathrm{D}$ modelling suggests that smooth inversion can achieve misfits very close to the minimum possible (HEINSON and CONSTABLE, 1992), so while we have no proof that a model that fits the COPROD2 data to RMS 1.0 or better does not exist, we consider the smooth inversion to be attaining close to the minimum possible misfit.

A number of reasons can account for our failure to fit the data. Firstly, the data errors could be systematically underestimated. For example, if the errors are half what they should be, then the errors reflect accurate relative weights and the residual variance of the inversion is a measure of the scaling required for the errors. Secondly, the effects of 3D structure in the data could be significant compared with data uncertainty. 3D effects could be in the form of large-scale structure (which would make 2D modelling a dubious exercise) or small-scale structure which varies from station-to-station but which is band-limited in the MT response functions. Such distortions of the MT data are sometimes called geological noise and can reasonably be treated as a stochastic process and incorporated into the data errors, although errors will necessarily then be correlated across adjacent frequencies. These distortions need not be large, as many of the data have errors as small as $1 \%$. That is, the data must reflect a purely $2 \mathrm{D}$ response to better than $99 \%$ before the original data can be fit using only a $2 \mathrm{D}$ model.

We accept the original data errors as a reasonable estimate of data repeatability. We assume that failure to fit the data is largely due to small-scale breakdown of the $2 \mathrm{D}$ assumption, and wish to increase the data errors to reflect this. Our concern is that a datum with a reproducability error of $1 \%$ but which cannot be fit to better than $10 \%$ because of $3 \mathrm{D}$ distortion of the impedance has a weight of 100 in a 2-norm measure of data misfit. Such a datum will have an enormous influence on the model. Because of this we choose a minimum error cutoff, such as $10 \%$, and any error below this is set to this value. Under the assumption that during the original response function estimation errors are partitioned equally between the real and imaginary components of the complex impedance, an error of $10 \%$ in apparent resistivity corresponds to $2.9^{\circ}$ in phase. Of course, 'geological' noise might not be partitioned this way, and indeed static shift as a manifestation of 3D structure has no effect on phase but a large effect on magnitude. However, we will be dealing with static shift explicitly, as well as checking our assumptions by examination of data residuals.

The inversion was applied to TE and TM apparent resistivity and phase data from 23 stations located over the NACP anomaly; stations PCS009 to PC5007 between $-124200 \mathrm{~m}$ and $117300 \mathrm{~m}$. Seven periods were chosen by taking every second period from the lower frequency part of the full COPROD2 data set, at 14, 28, 57, 114, 228, 455 and $910 \mathrm{~s}$. These data have been previously corrected for static shift (JONES, 1988), and the longest period for station PC5007 was flagged as bad. All four data from this site for this period have been omitted, giving a total of 640 independent data. Previous inversions of this data set using all stations and six periods (DEGroot-Hedlin and Constable, 1990; DeGroot-Hedlin, 1991) indicates that error thresholds of $10 \%$ in resistivity and $2.9^{\circ}$ in phase are indeed necessary to fit the data; larger values resulted in the blurring of significant structure.

The model was initially constructed to have a total of 21 layers, with a top layer $600 \mathrm{~m}$ thick and 10 layers per decade below this. In the horizontal direction, columns were arranged so that station locations were in the centres of the conductivity bricks. Additional columns were added as necessary to prevent column widths from exceeding $10 \mathrm{~km}$. This produced 39 bricks in the horizontal direction at the surface. Deeper in the model adjacent bricks are combined in order to keep the aspect ratio close to one. After 15 iterations starting from a half-space conductivity of $100 \Omega \mathrm{m}$ the inversion was stopped. Two more layers were added to the bottom of the mesh to avoid a sharp jump in resistivity that had developed at the terminating layer. The upper 6 layers 
were also adjusted in thickness to correspond with a 1D inversion of the high frequency data being run simultaneously (discussed below), in anticipation of constraining these layers to agree with the $1 \mathrm{D}$ conductivities. The resulting model had 764 parameters and $89 \times 30$ finite element nodes, with 2 horizontal and 2 vertical nodes in the parameter bricks of the upper 2 layers and 2 horizontal and 1 vertical node beneath this. Such a sparse finite element mesh is possible because the models are forced to be smooth. The inversion was continued to convergence, and the final model fitting the TE and TM apparent resistivity and phase to RMS 1.0 is shown in the upper frame of Fig. 1.

The normalized residuals corresponding to the joint TE/TM model are plotted in Fig. 2. These residuals $r$ are calculated as

$$
r_{i}=\frac{d_{i}-d_{i}^{\prime}}{\sigma_{i}}
$$

where $d_{i}^{\prime}$ is the $i^{\text {th }}$ model response, $d_{i}$ is the $i^{\text {th }}$ datum, and $\sigma_{i}$ is the associated error. Assuming that errors in the data are due to a zero-mean Gaussian process, the weighted data misfits should be randomly distributed and uncorrelated with space and period. The presence of trends in the residuals would indicate either a bias in the inversion routine, a systematic mis-estimation of errors, or failure of the assumptions used in constructing the model. The first of these has been ruled out based on tests of the inversion routine on synthetic data (DEGROOT-HEDLIN and CONSTABLE, 1990). In spite of the large difference in the amount of structure exhibited between the TM and TE modes data, the misfit is partitioned very well between the two modes, as RMS 1.016 and 0.984 respectively (the expected value plus or minus one standard deviation is RMS 1.03 to 0.97 ). Similarly, misfit partitioning between apparent resistivity and phase is almost perfect at RMS 1.002 and 0.998 respectively. However, as is evident from the figure, the misfit is worse at long periods than at short periods. The misfit for the 4 highest frequencies compared to the 3 lowest frequencies is 0.65 versus 1.34 . The long-period data are indeed more noisy than the shorter periods, and this has not been accommodated by our simple $10 \%$ threshold because even though larger, the long-period errors are generally still less than that threshold. Alternatively, SMITH and BOOKER (1988) point out that if an excessive penalty is placed on the deeper structure compared with shallower structure then this sort of behaviour will be observed. They call it a 'coloured' fit. However, the penalty structure of the 2D implementation of OCCAM is specifically designed to avoid this bias by decreasing the penalty exponentially with depth, and does not produce coloured fits on synthetic test cases (DEGroot-Hedlin and Constable, 1990). The model itself is 'coloured', with most structure occurring between $8 \mathrm{~km}$ and $25 \mathrm{~km}$ depth and confined laterally to the central third of the model, but this structural pattern does not explain the larger residuals at long periods and all station locations.

Besides partitioning of the error budget, one is concerned about large blocks of data being underfit or overfit (large patches of green or red in the figure). This is either due to (i) underfitting the data, (ii) underparameterization of the model, or (iii) lack of independence of data errors. Experience suggests that better fits to the data are difficult to obtain and result in unreasonably large extremes of conductivity. Since the 2D mesh is moderately fine, underparameterization in this case basically means $3 \mathrm{D}$ structure that is not represented by a $2 \mathrm{D}$ model. Ideally the data are independent from site to site with no coherent noise, such as might arise from source-field effects. The slight tendency for site-to-site correlation, more evident in the TM mode data, is presumably caused by a breakdown of the $2 \mathrm{D}$ approximation that extends over adjacent sites. The tendency for residuals of about 2 adjacent periods to group is likely to be caused by correlated data errors: It is not unusual for the time-series analysis used to generate response functions to have frequency resolution that is worse than the frequency spacing of the data, producing data that are not truely independent between adjacent frequency bands. There is also a systematic underfitting of the 2 or 3 highest frequency TM resistivity data and 2 or 3 middle frequencies of 
the TM phase. However, on the whole the data are fit very well and there are no serious trends in the residuals.

Near-surface structure (above $3 \mathrm{~km}$ ) is poorly resolved by the data at these periods, but there is a marked tendency to make columns between stations more conductive. The NACP anomaly appears as a complex structure near the centre of the profile, consisting of three conductive regions increasing in depth from about $8 \mathrm{~km}$ in the east to about $22 \mathrm{~km}$ in the west, set in a fairly resistive background of 100 to $1000 \Omega \mathrm{m}$. The conductive bodies are moderately elongate in a vertical direction and do not affect the east-west currents sufficiently to produce an anomalous TM response, thus the insensitivity of the TM data (JONES and SAVAGE, 1986) to this feature is reproduced by the model. The lowest resistivity of the westernmost conductive feature is $0.17 \Omega \mathrm{m}$. This is an unusually low value for resistivity, but although smooth inversion may overshoot the peak conductivity of conductive prisms (DEGROOT-HEDLIN and CONSTABLE, 1990), this low resistivity is consistent with the 0.2-0.3 $\Omega \mathrm{m}$ of JONES and CrAVEN (1990). Hot brines might be responsible for such low resistivities, but high porosities are unlikely at these depths. Graphitic carbon could easily account the resistivities this low (DUBA and SHANKLAND, 1982) and is considered to be present in granulite facies rocks from the mid- to lower-crust (e.g. MARESCHAL et al., 1992).

While three discrete bodies clearly fit the data, it is possible that the same effect on the TE and TM modes could be obtained if the rocks were anisotropic, or foliated in the vertical direction (DEGROOT-HEDLIN, 1991); the smooth model must represent such structure by modelling anisotropic fabric as a macro-anisotropic structure similar to that seen in Fig. 1. If this were the case the conductivity in the vertical and north-south directions would be lower than the peak value modelled, or about $1 \Omega \mathrm{m}$, and the conductivity in the east-west direction would be similar to the 100 to $1000 \Omega \mathrm{m}$ background resistivity. DEGROOT-HEDLIN (1991) made preliminary efforts to test these ideas by modelling a single, anisotropic body. Such a feature did indeed fit the data and resulted in a slight improvement in distribution of residuals.

Vertical field data are available as part of the COPROD2 data set and were examined by DEGroot-HedLIn (1991). These data are extremely noisy and the error threshold had to be increased to $60 \%$ before an adequate fit could be obtained. The TE apparent resistivities from one station were included in the inversion to constrain the average resistivity of the model. The vertical field inversion produced a single conductive body that was more diffuse than those of the resistivity and phase inversion. However, the feature was in the location expected from the inversion shown in Fig. 1. Because of this large error level and basically compatible structure, it is not likely that inclusion of the vertical field in the TE/TM inversion would change the resulting models significantly.

The iterative refinement of data error structure and model parameterization described above is typical when applying the Occam process to a complicated problem such as 2D MT. That is, some experimentation is needed to find an appropriate level for fitting the data and further experimentation is required to find the depths of resolution that need to be represented in the model. However, in order to illustrate the convergence properties of the algorithm, the inversion was repeated three more times from starting half-spaces of 10,100 and $1000 \Omega \mathrm{m}$, using the final model parameterization and data errors derived above. The stepsize, model roughness and misfit at each iteration are shown in Fig. 4. The desired fit to the data was obtained in 9-10 iterations but it takes at least 3 more iterations to remove excess structure in the model. The stepsize should decrease to zero as the inversion converges, and does in the case of 1D MT inversions. However, for the $10 \Omega \mathrm{m}$ starting model the inversion was carried to 31 iterations, and we see the stepsize asymptote to about 0.4 as small amounts of structure continues to be removed from the model. We suspect that limitations in the accuracy of the forward code, particularly in the computation of derivatives, is responsible for this behaviour.

The inversion starting from $100 \Omega \mathrm{m}$ has the least structure after 16 iterations and is essentially 

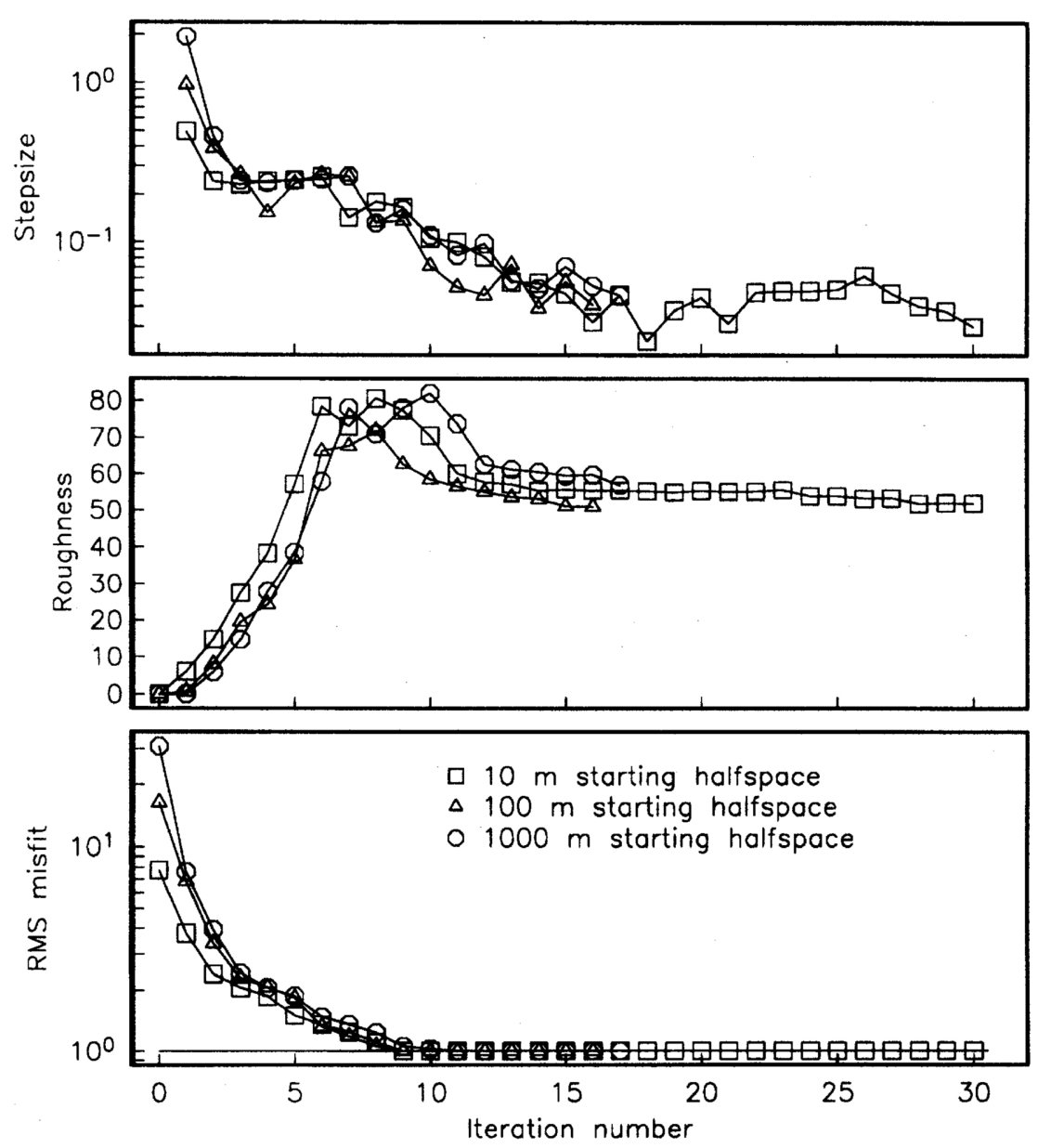

Fig. 4. The size of the model step, model roughness, and root-mean-square data misfit as a function of iteration number for three different starting models. The horizontal line indicates the desired misfit of RMS 1.0.

identical to the one shown in Fig. 1. The other two inversions developed conductive features at the surface of the model at the expense of the third, easternmost, element of the NACP anomaly. The surface features were between stations and represent an extreme case of the tendency, already noted, to make any surface blocks not constrained by a station more conductive. We suspect that this is a result of the station spacing being comparable to the depth of the shallow parts of the NACP conductor. In the following section we describe an attempt to constrain the surface structure using shorter-period data. There seems to be a dependence on starting model for the $2 \mathrm{D}$ inversion that is not seen in $1 \mathrm{D}$ inversions. It is generally more difficult to remove unnecessary structure, and starting models other than half-spaces pose more difficulty for the inversion algorithm in both $1 \mathrm{D}$ and $2 \mathrm{D} \mathrm{MT}$ problems. It appears that in $2 \mathrm{D} \mathrm{MT}$ inversion, if conductive structure develops in the wrong part of the model it is difficult to remove, because of the large effect this structure has on the derivatives. For example, if conductive surface structure appears then the MT response is not sensitive to deeper structure, and the sensitivity functions, or rows of the Jacobian matrix, peak within the conductive feature. We do not know that, given 
a large number of iterations, the surface features would not migrate deeper into the model, but such a large number of iterations is clearly impractical.

\section{Inversion of Short Period Data}

In this section we use the short period raw data, which has not been corrected for static shifts, to solve for the shallow resistivity structure and static shifts simultaneously. As previously mentioned, structure resolved by short period data has negligible effect on the responses at neighbouring stations. Therefore, in order to build a $2 \mathrm{D}$ model of the shallow structure using these periods, reasonable constraints must be imposed upon the model of the shallow structure. For instance, the assumption of a layered conductivity structure beneath each site is a reasonable one in this case because the short period COPROD2 data suggest that the near surface conductivity structure is approximately 1D (JONES and SAVAGE, 1986). In order to treat these data in a computationally efficient manner, we introduce a modification to the Occam inversion algorithm.

For the modified inversion method, the model is composed of a column of blocks of uniform resistivity under each station. The model responses and derivatives are found by treating the column of blocks beneath each station as a set of layers and calculating the corresponding 1D responses. The calculation of the derivatives for the $1 \mathrm{D}$ problem is described in CONSTABLE et al. (1987). One could use this method of constructing a 2D model based on 1D approximations for any range of periods to solve for a model approximately fitting the data, using the usual roughness measure in which the horizontal penalty is set equal to the aspect ratio of each individual parameter block (DEGROOT-HEDLIN, 1991). Such a penalty ensures that the model is not biased in favour of either vertically or horizontally elongated structures. However, for the inversion of the short period data in the period range up to $7.1 \mathrm{~s}$, the penalty on the horizontal roughness was increased in order to make the shallow structure more 1D. This proved necessary for a stable solution of the static shifts since static shifts serve largely to reduce the horizontal roughness of the model. Without this modification, the vertical dimensions of the blocks are constrained by the small skin depths for these periods, while the horizontal dimensions of the blocks are given by the distances between stations, about $10 \mathrm{~km}$ on average. The resulting horizontal roughness penalty is small compared to the penalty for vertical roughness since the aspect ratio is very small. Thus, in effect, a series of $1 \mathrm{D}$ inversions is performed at each station since there is little constraint on the lateral roughness of the model. This approach results in little constraint on the static shift values.

The following approach, using two Lagrange multipliers, proved to be a robust means of forcing horizontal smoothing. The functional to be minimized is given by

$$
U_{1}[\mathbf{m}]=\lambda\left\|\boldsymbol{\partial}_{y} \mathbf{m}\right\|^{2}+\mu\left\|\boldsymbol{\partial}_{z} \mathbf{m}\right\|^{2}+\left\{\left\|\mathbf{W}\left(\mathbf{d}-F_{1}(\mathbf{m})\right)\right\|^{2}\right\}
$$

where $\lambda$ and $\mu$ are the Lagrange multipliers, and $F_{1}$ is the 1D forward functional which is linear with respect to the static shifts and nonlinear with respect to the resistivities. In the standard approach (Eq. (1)) $\lambda=\mu$, but this formulation allows the relative weight between the vertical and horizontal roughness to be controlled by the Lagrange multipliers. Increasing $\lambda$ over $\mu$ is equivalent to increasing the penalty on the horizontal roughness over that of the vertical roughness. At each iteration a stationary point for $U_{1}$ is given by

$$
\mathbf{m}(\lambda, \mu)=\left[\left(\lambda \boldsymbol{\partial}_{y}^{T} \boldsymbol{\partial}_{y}+\mu \boldsymbol{\partial}_{z}^{T} \boldsymbol{\partial}_{z}\right)+\left(\mathbf{W} \mathbf{J}_{1}\right)^{T} \mathbf{W} \mathbf{J}_{1}\right]^{-1}\left(\mathbf{W} \mathbf{J}_{1}\right)^{T} \mathbf{W} \hat{\mathbf{d}}
$$

where $\mathbf{J}_{1}$ is the Jacobian matrix of partial derivatives of $F_{1}$ with respect to the model. The values of Lagrange multipliers $\lambda$ and $\mu$ must be determined in each iteration. During the initial iterations, which take the (featureless) starting model to a feasible model (i.e. one fitting the 
data), we set $\lambda=\mu$ and a univariate search is conducted along $\mu$ to find a model minimizing the misfit to the data. Once the mimimum misfit at an iteration is less than the required misfit, the Lagrange multiplier $\mu$ is held fixed and $\lambda$ is increased, increasing the horizontal smoothness, until the desired misfit is attained. Convergence is reached once the horizontal roughness no longer decreases from one iteration to the next. The result is a model exhibiting little horizontal structure; we call this the quasi-1D model. This modified Occam method is an efficient, as well as accurate, means of inverting the short period COPROD2 data, because the computation time for the $1 \mathrm{D}$ responses and derivatives is insignificant compared to those for the fully $2 \mathrm{D}$ calculations.

Like the conductivity structure, the static shift part of the model will depend on the tradeoff between data fit and model penalty, and therefore an accurate determination of the data quality is necessary. The $\mathrm{D}^{+}$model (PARKER, 1980; PARKER and WHALER, 1981) may be used to test the validity of the $1 \mathrm{D}$ assumption for a given data set and to find the minimum possible misfit. One would not expect a joint application of the $\mathrm{D}^{+}$inversion to the original TE and TM data to yield a $1 \mathrm{D}$ model, as static shifts remain in the data. We therefore applied the $\mathrm{D}^{+}$algorithm separately to each of the TE and TM modes for fifteen periods in the range $.056 \mathrm{~s}(18 \mathrm{~Hz})$ to $7.1 \mathrm{~s}$ at each of 34 stations, with the minimum error in the data set to $5 \%$. The resulting RMS misfits varied between 0.32 and 1.72 , with a mean of 0.72 . It appears, based on experience and simulations such as undertaken by HEINSON and CONSTABLE (1992), that if the expected misfit value is 1.0 then the $\mathrm{D}^{+}$misfit will be around 0.8 . Thus a minimum error of $4 \%$ to $5 \%$ for a TE or TM-only inversion of the short period data appears reasonable. When combining TE and TM information one would expect the error to be greater by a factor of approximately $\sqrt{2}$ because the $\mathrm{D}^{+}$model is fitting noise in each dataset independently, so the minimum misfit for the combined $\mathrm{TE} / \mathrm{TM}$ data in the range .056 to $7.1 \mathrm{~s}$ was set at $6 \%$.

The modified Occam algorithm was applied to the joint TE/TM data set having a minimum error of $6 \%$. Fifteen periods in the range 0.056 to $7.1 \mathrm{~s}$ were used at 34 stations (station PCSE04 was omitted since these raw data were unavailable). The quasi-1D model, fitting this data set to RMS 1, was attained after 3 iterations and is shown in Fig. 3. The locations of the lateral boundaries of the columns are fairly arbitrary, as the blocks are treated as layers in the forward problem, but are taken to be halfway between the adjacent stations. The data allow a highly 1D model in the top $4 \mathrm{~km}$, with a conductive layer sandwiched between two more resistive layers. The conductive layer becomes shallower going from west to east. The static shift parameters derived in this inversion are shown in Fig. 5, with the shifts derived in JONES (1988) shown for comparison. Since Jones used a net downward shift for his data, his static shifts have all been shifted upward by 0.076 in order to compare them with the static distortions derived here, which sum to zero. The assumptions used here are similar to those used by Jones, and so the close agreement between the two sets of static shifts is not surprising, but the method of calculating static shifts presented here is automatic and does not rely on detailed knowledge of the subsurface. Clearly, a full 2D forward calculation of the responses for the model in Fig. 3 is unnecessary and the 1D approach gives highly accurate results. The residuals (DEGROOT-HEDLIN, 1991) indicate that the misfit is partitioned well between the TE and TM modes. No serial correlation is evident, indicating that an approximately 1D model is valid for this period range. Furthermore, a Kolmogorov-Smirnoff test indicated that the distribution of the weighted residuals is indistinguishable from a Gaussian distribution, further suggesting that data in this period range are minimally affected by $2 \mathrm{D}$ or $3 \mathrm{D}$ structure.

\section{Fitting Both Short and Long Period Data}

The surface conductivities derived from the 1D inversion described above are higher than in the long-period model shown in Fig. 1, and we wish to incorporate them into the long-period model. It is evident from Fig. 3 that the number of layers in the shallow model is much greater 

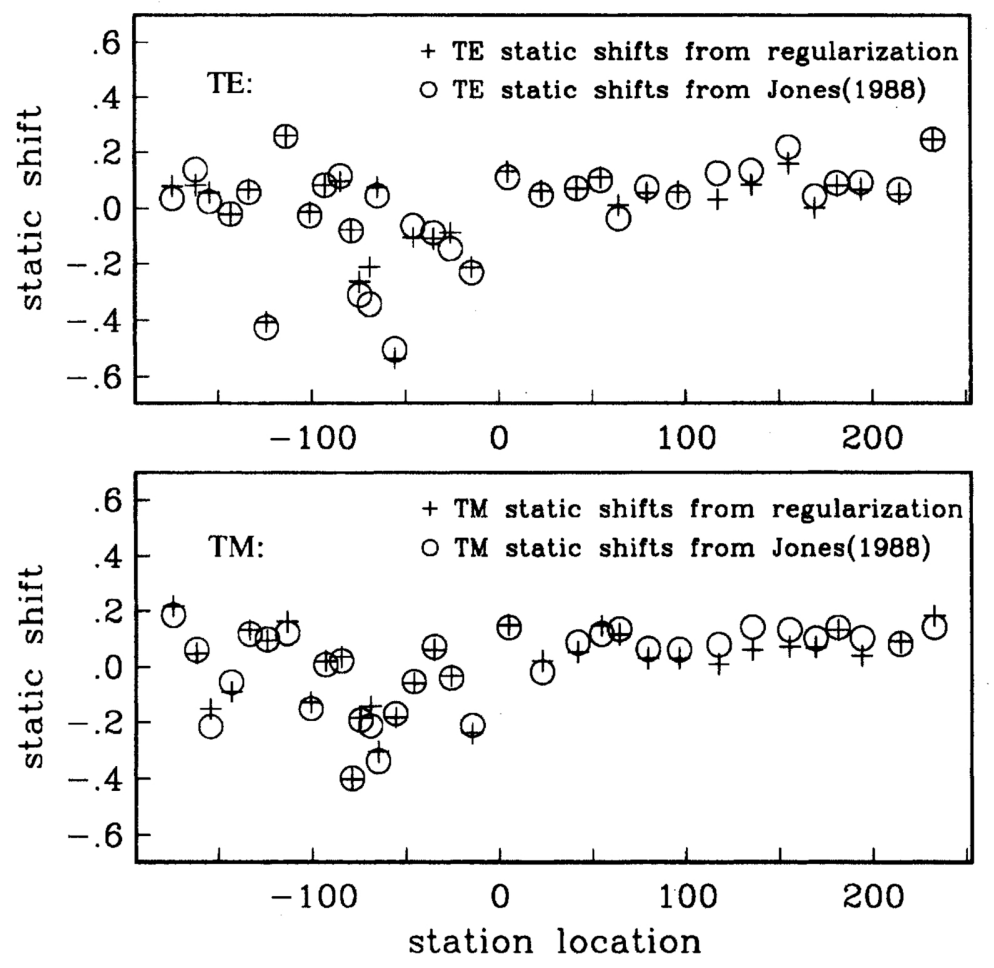

Fig. 5. The static shifts derived from the psuedo-1D modelling compared to those of JonES (1988). A net bias in the static shifts cannot be resolved by this method, and so Jones' static shifts were translated upward by 0.076 for the purpose of comparison.

than it needs to be. The thicknesses of the upper 7 layers of the long-period model and the layering of the quasi-1D model were both adjusted to match the dominant features portrayed in Fig. 3. Also, given that our static shifts agree well with Jones' and the desire for consistency with other studies published in this volume, we used the static shifts derived by JonEs (1988). The quasi-1D inversion was re-run using these new layer thicknesses and the fixed static shifts.

There are various ways to incorporate the shallow structure into the long-period inversion:

A) We could construct a hybrid forward modelling algorithm which uses $2 \mathrm{D}$ computations for the long periods and 1D computations for the short. Since the thickness of the surface layers in the long-period model has been set to match the surface structure from the 1D modelling, this does not increase the number of parameters but does increase the size of $\mathbf{d}$ and $\mathbf{J}$.

B) The surface structure in the long-period model can be held fixed at the values derived from the $1 \mathrm{D}$ modelling, and the uppermost layer of the deeper structure penalized against the deepest layer of the shallow (1D-derived) structure. How one accomplishes such a penalty in practice is explained below. This approach decreases the size of $\mathbf{m}$ and $\mathbf{J}$, but forces the $2 \mathrm{D}$ inversion to adopt the $1 \mathrm{D}$-derived, shallow structure whether the high and low frequency data are mutually compatible or not.

C) The shallow, 1D-derived structure can be made preferred, but not fixed, in the 2D inversions of the long-period data (again, this is explained below). This does not alter the size of the inversion, but does complicate the penalty measure on the model. This is the approach we will take in this paper. 
In order to penalize a boundary between the free parameters and fixed conductivity structure, or include preferred conductivity structure, another term must be included in the unconstrained functional;

$$
U=\|\boldsymbol{\partial} \mathbf{m}\|^{2}+\|\mathbf{T}(\mathbf{m}-\mathbf{p})\|^{2}+\mu^{-1}\|\mathbf{W}(\mathbf{d}-F(\mathbf{m}))\|^{2} .
$$

The term involving $\mathbf{T}$ serves to minimize the horizontal and vertical differences between the model vector $\mathbf{m}$ and vector of known values $\mathbf{p}$. The diagonal matrix $\mathbf{T}$ relating the model $\mathbf{m}$ to fixed parameters $\mathbf{p}$ has non-zero values on the diagonals corresponding to the indices $i$ at which we wish to minimize $\left(m_{i}-p_{i}\right)$, and zeroes elsewhere.

Because $\mathbf{T}$ is symmetric the stationary point for $U$ may be written as

$$
\mathbf{m}(\mu)=\left[\left(\mu\left(\boldsymbol{\partial}^{T} \boldsymbol{\partial}+\mathbf{T} \mathbf{T}\right)\right)+\left(\mathbf{W} \mathbf{J}_{2}\right)^{T} \mathbf{W} \mathbf{J}_{2}\right]^{-1}\left[\left(\mathbf{W} \mathbf{J}_{2}\right)^{T} \mathbf{W} \hat{\mathbf{d}}+\mu \mathbf{T} \mathbf{p}\right] .
$$

In general, this formulation may be used to force model parameters close to some preferred resistivity values designated by the vector $\mathbf{p}$. To use this formulation to smooth a boundary between fixed and variable model parameters, the vector $\mathbf{p}$ is composed of the fixed model values of blocks which lie next to a contact between the fixed and free model parameters. These values are ordered so that the indices of the fixed values correspond to the indices of the adjacent free parameters. The nonzero values of the matrix $\mathbf{T}$ are located on rows corresponding to these same model indices and the scalar values are given by the weights which would appear in the appropriate penalty matrix at this location. The remaining values of $\mathbf{p}$ are acted upon by rows of zeroes in the matrix $\mathbf{T}$ and therefore do not need to be specified.

We included the upper 5 layers (a total thickness of $2.2 \mathrm{~km}$ ) of the quasi-1D model as preferred structure in the inversion of the long-period data set used before. This inversion was much slower to converge, probably because the complexity of the penalty term is increased, with the resistivities of the upper 5 layers being smoothed against each other as well as being penalized for differing from the preferred structure. Also, it was more difficult to fit the data. The long-period data are not necessarily compatible with the short-period data used to generate the preferred structure and we are, in effect, using a much broader spectrum of data in the inversion. So, although very rough models fitting to RMS 1.0 could be obtained, we relaxed the acceptable level of misfit to $15 \%$, and ran the model to convergence. The result is shown in the lower frame of Fig. 1, and differs from the previous model in several ways. The surface structure is, as expected, more conductive and closer to the values obtained from the inversion of the higher frequency data. (Details of features seen in Fig. 3 are lost because of the coarser parameterization and much expanded resistivity scale.) The NACP anomaly has evolved from three discrete prisms to a more complex conductive feature having an upper surface which is more constant in depth (at about 12-15 km) and a lower surface that extends much deeper (to about $75 \mathrm{~km}$ ) and is, perhaps, connected to the mantle. Background resistivities of the basement crustal rocks are higher, extending to $10,000 \Omega \mathrm{m}$ in places, and the boundary between the surface sediments and the basement is much sharper. Upper mantle resistivities are lower. Most intriguing, however, is the development of a persistent westward dipping structure in the crustal basement below $10 \mathrm{~km}$ depth. This structure suggests that the NACP anomaly is only an unusually conductive example of a more pervasive geological fabric.

The inclusion of the surface sediment conductivities makes this model more realistic but is perhaps forcing an over-fitting of the long-period data. The residuals are systematically positive at the shortest period or two, suggesting that the high and low frequency data are not easily reconciled. A resolution analysis, to examine the reliability of this model, is beyond the scope of the current paper. However, the features in this model are not geologically unreasonable, and are perhaps more realistic than the three separate, simple prisms of the first model. We include this inversion as an example of how the simple $2 \mathrm{D}$ algorithm can be extended to include a prejudice for model parameters. 


\section{Conclusions}

We have demonstrated that regularized inversion, implemented via the Occam's inversion algorithm, is a stable and versatile scheme for the interpretation of approximately 2D MT data sets. Current computer technology makes this approach viable using of order 1000 data components and order 1000 model parameters. Since the Occam algorithm is completely general, being decoupled from the forward problem, substitution of other methods of calculating forward responses and the Jacobian matrix of derivatives is possible. In particular, we demonstrated the use of 1D. forward responses and derivatives to generate models fitting the predominantly 1D short-period data. Furthermore, we introduced a modification to the basic algorithm that allowed penalties to be applied against fixed or preferred structure in the model. For the COPROD2 data, this was useful for incorporating shallow structure, derived from inversions using a 1D approximation, into the long-period (from $14 \mathrm{~s}$ to $910 \mathrm{~s}$ ) inversions for deep structure.

We have been able to fit the COPROD2 data to a root-mean-square average of $10 \%$ in apparent resistivity and $2.9^{\circ}$ in phase. Since these values are considerably larger than the measurement errors supplied with the data, data errors were truncated at a minimum of these uncertainty levels. We have argued that an assessment of the inverted models must include an examination of the weighted data residuals. In this case the residuals are very evenly and randomly distributed between the various modes (TE and TM resistivity and phase) but with a slight tendency for larger misfits in the long period data than in the short period data. The model thus obtained features three distinct conductive prisms at the position of the NACP anomaly. A model in which surface structure derived from 1D inversion of the highest frequency data was included as preferred structure shows considerably more complexity in the basement structure, including a westward dipping fabric in the lower crust between $10 \mathrm{~km}$ depth and the mantle.

The authors thank Alan Jones for providing the COPROD2 data and assisting in early attempts to apply the Occam algorithm to them; his consistent support of this project is appreciated. The authors thank Phil Wannamaker for supplying the forward code essential to the 2D MT implemention of Occam, John Booker for pointing out an error we had made in error partioning, Pete Savage and PanCanadian for making these data available, and two anonymous reviewers. WSE Associates supplied support for improving the OCCAM2DMT code. Part of this work was conducted under support from the NSF by grant EAR8916958 and the Scripps block grant to the San Diego Supercomputer Center.

\section{REFERENCES}

Alabi, A. O., P. A. Camfield, and D. I. Gough, The North American Central Plains anomaly, Geophys. J. R. astr. Soc., 43, 815-834, 1975.

Camfield, P. A. and D. I. Gough, A possible Proterozoic plate boundary in North America, Can. J. Earth Sci., 14, 1229-1238, 1977.

Constable, S. C., R. L. Parker, and C. G. Constable, Occam's inversion: a practical algorithm for generating smooth models from EM sounding data, Geophysics, 52, 289-300, 1987.

DEGroot-Hedlin, C. D., Removal of static shift in two dimensions by regularized inversion, Geophysics, 56, 2102-2106, 1991a.

DEGRoot-HedLin, C. D., Occam2: Regularized inversion to generate smooth, two-dimensional resistivity models from magnetotelluric data, Ph.D. Dissertation, Univ. Calif. San Diego, 1991b.

DEGroot-Hedlin, C. D. and S. C. Constable, Occam's inversion to generate smooth, two-dimensional models from magnetotelluric data, Geophysics, 55, 1613-1624, 1990.

Duba, A. and T. J. Shankland, Free carbon and electrical conductivity in the Earth's mantle, Geophys. Res. Lett., 9, 1271-1274, 1982.

Heinson, G. S. and S. C. Constable, The electrical conductivity of the oceanic upper mantle, Geophys. J. Int., 110, 159-179, 1992.

Jones, A. G., Static shift of magnetotelluric data and its removal in a sedimentary basin environment, Geophysics, 53, 967-978, 1988. 
Jones, A. G. and P. J. Savage, North American Central Plains conductivity anomaly goes east, Geophys. Res. Lett., 13, 685-688, 1986.

Jones, A. G. and J. A. CrAven, The North American central plains anomaly and its correlation with gravity, magnetic, seismic, and heat flow data in Saskatchewan, Canada, Phys. Earth Planet. Inter., 60, 169-194, 1990.

Mareschal, M., W. S. Fyfe, J. Percival, and T. Chan, Grain-boundary graphite in Kapuskasing gneisses and implications for lower-crustal conductivity, Nature, 357, 674-676, 1992.

PARKER, R. L., The inverse problem of electromagnetic induction: existence and construction of solutions based upon incomplete data, J. Geophys. Res., 85, 4421-4425, 1980.

PARKER, R. L., The inverse problem of resistivity sounding, Geophysics, 49, 2143-2158, 1984.

PARKer, R. L. and K. A. Whaler, Numerical methods for establishing solutions to the inverse problem of electromagnetic induction, J. Geophys. Res., 86, 9574-9584, 1981.

Sмiтh, J. T. and J. R. Booker, Magnetotelluric inversion for minimum structure, Geophysics, 53, 1565-1576, 1988. 